\title{
A Feasibility Study of RIP Using 2.4 GHz 802.15.4 Radios
}

\author{
B.J.Dil ${ }^{1}$, P.J.M.Havinga ${ }^{2}$ \\ Pervasive Systems, University of Twente \\ Zilverling building, 7522 NB Enschede, The Netherlands \\ ${ }^{1}$ B.J.Dildutwente.nl \\ ${ }^{2}$ P.J.M.Havinga@ewi.utwente.nl \\ Ambient Systems \\ Colosseum 15d, 7521 PV Enschede, The Netherlands \\ ${ }^{1}$ Brameambient-systems. net
}

\begin{abstract}
This paper contains a feasibility study of Radio Interferometric Positioning (RIP) implemented on a widely used $2.4 \mathrm{GHz}$ radio (CC2430). RIP is a relatively new localization technique that uses signal strength measurements. Although RIP outperforms other RSS-based localization techniques, it imposes a set of unique requirements on the used radios. Therefore, it is not surprising that all existing RIP implementations use the same radio (CC1000), which operates below the $1 \mathrm{GHz}$ range. This paper analyzes to what extent the CC2430 complies with these requirements. This analysis shows that the CC2430 platform introduces large and dynamic sources of errors. Measurements with a CC2430 test bed in a line-of-sight indoor environment verify this. The measurements indicate that the existing RIP algorithm cannot cope with these types of errors, and will incur a relatively low accuracy of 3.1 meter. Based on these results, we made an initial implementation of a new algorithm, which can cope with these errors, and decreases this positioning error by a factor of two to 1.5 meter accuracy.
\end{abstract}

\section{INTRODUCTION}

This paper focuses on localization in static wireless networks. Localization in these networks describes the process of obtaining a physical location in an automated manner using wireless communication. Many wireless network applications rely on location information to perform their tasks. Locations provide context to the measured data (e.g. like measuring temperature); localization can be a stand-alone application (e.g. inventory tracking in a distribution center) or provides support to the network service (e.g. routing). Today, such applications have evolved into real-time location systems (RTLS) using a wide range of wireless technologies. Many of these localization applications are based on Received Signal Strength (RSS) measurements, as RSS information is obtained without additional hardware and energy costs. Other localization systems use techniques like Time Difference Of Arrival (TDOA), Time Of Flight (TOF), Ultra Wide Band (UWB) and Angle Of Arrival (AOA). In general, these techniques are more accurate than RSS-based localization but require specialized hardware, more processing, more communication and thus more energy (e.g. [1]). Radio Interferometric Positioning holds the promise to break this paradigm.
This paper contains a feasibility study on Radio Interferometric Localization within the $2.4 \mathrm{GHz}$ range implemented on a widely used radio (CC2430, [12]). Radio Interferometric Localization relies on a pair of nodes simultaneously transmitting unmodulated carriers at slightly different frequencies. Nodes within transmission range measure the energy of the envelope of the composite signal. The relative phase offset of the measured signal amplitudes at two receivers is a function of the distances between the involved nodes and the carrier frequency. This information is used to estimate the position of the nodes ([2]).

The main challenge of the current implementation of RIP is that it requires the radio to tune its frequency in fine-grain steps, such as the CC1000 (see [11], 65 Hertz frequency resolution). Most Commerical-Off-The-Shelf (COTS) radios cannot comply with this requirement, like the CC2430 ([12]). Moreover, all existing implementations use the CC1000 (e.g. [2]). This paper focuses on RIP implementations when the radios can not comply with this requirement. As an example, we implement RIP on a CC2430 platform.

We see the main contributions of this paper as:

- It analyzes the errors introduced by the radios that can not sufficiently fine tune its frequency. We show that these errors ALL depend on the accuracy of the used crystal oscillator. Moreover, this analysis shows that this new radio platform introduces large and dynamic sources of error.

- We implement and evaluate RIP on a new radio platform. These measurements show that the RIP does not provide the required results $(\sim 3.1$ meter $)$. Moreover, an initial implementation of a new algorithm decreases this positioning error to 1.5 meter accuracy.

This paper is organized as follows. After a short introduction to RIP and overview of existing work in Section II, we analyze the errors introduced by the radios. Section IV analyzes the errors introduced by the existing and new implementation strategy. Section V describes the measurement set-up and evaluates the performance of the existing RIP algorithms. 


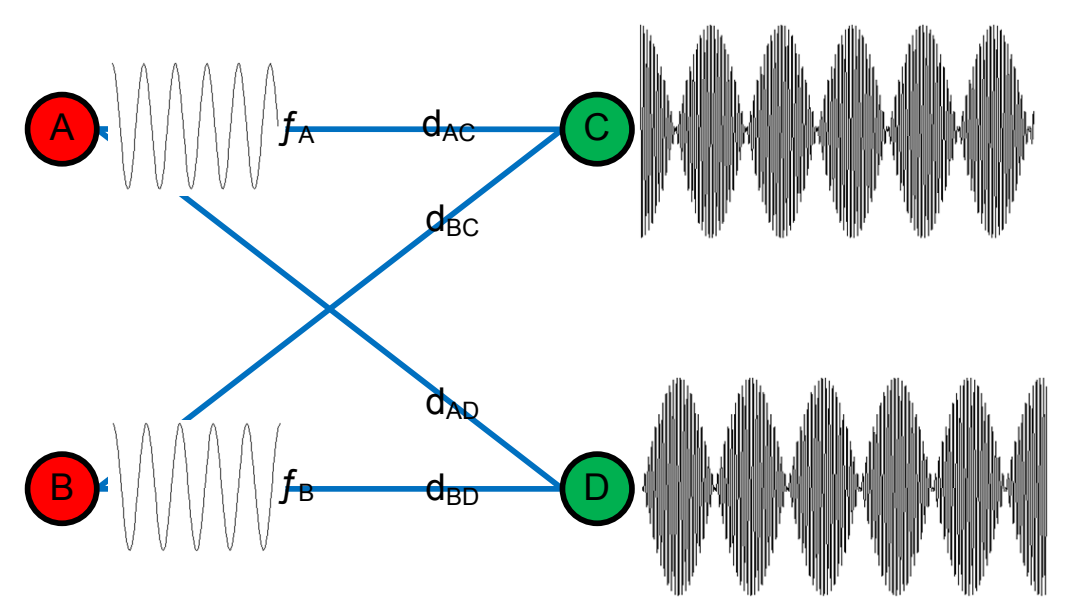

Fig. 1. RIP Measurements

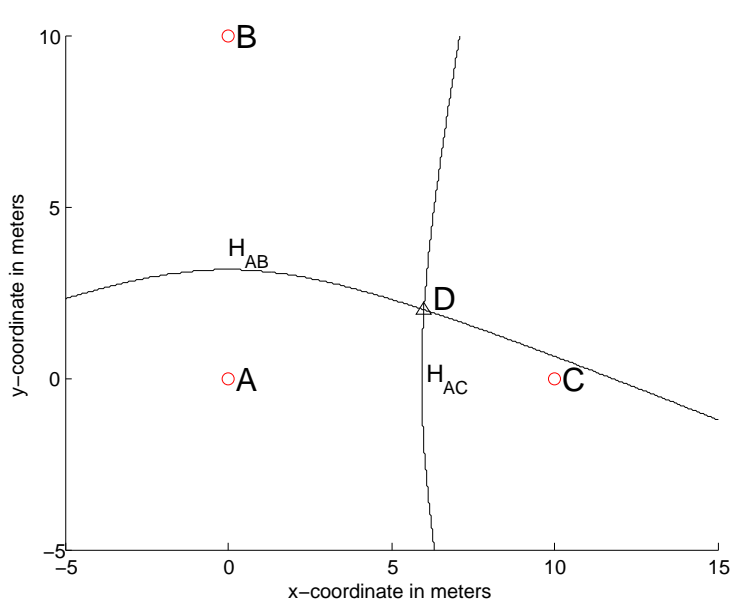

Fig. 2. Localization using hyperbolic curves
Section VI gives a conclusion.

\section{BACKGROUND AND RELATED WORK ON RADIO INTERFEROMETRIC POSITIONING}

This section contains a short description of the RIP technique. For a more detailed description we refer to [2]. Moreover, this section relates this short description to related work in this field. Figure 1 shows an example of a RIP set-up. Here nodes $A$ and $B$ transmit an unmodulated carrier signal with frequencies $f_{A}$ and $f_{B}$. The envelope of the composite signal of $A$ and $B$ has a beat frequency of $\left|f_{A}-f_{B}\right|$, which is measured by node $C$ and $D$. Figure 3 shows an illustrative example of the measured composite RSSI signal over time (frequency beat). The relative phase offset of the measured beat frequency is a function of the distances between nodes $A / B / C / D$, assuming that $f_{A}>f_{B}$ :

$$
\Delta \varphi_{i}=2 \pi \frac{d_{A B C D}}{\lambda_{i}} \bmod (2 \pi)
$$

Here:

$$
d_{A B C D}=d_{A D}-d_{B D}+d_{B C}-d_{A C}
$$

Here $\Delta \varphi_{i}$ represents the relative phase offset; $d_{A D}$ represents the distance between node $A$ and $D ; \lambda_{i}$ represents the wavelength of the intermediate frequency: $\frac{c}{f_{i}}=\frac{2 c}{f_{A}+f_{B}}$. Here $c$ represents the speed of light; and $f_{i}$ represents intermediate frequency $i$. We define $d_{A B C D}$ as the q-range, as in [3].

Due to $(\bmod (2 \pi))$-related ambiguity of $d_{A B C D}$, Equation 1 does not define a unique solution for $d_{A B C D}$. Existing RIP implementations solve this problem by performing measurements over several frequencies: $f_{1} \ldots f_{N}$. Then the problem can be rewritten to the following optimization problem ([3]):

$$
\operatorname{ERROR}\left(d_{A B C D}\right)=\sum_{i=1}^{N}\left(d_{A B C D}-d_{i}\right)^{2}
$$

Here ([3]):

$$
d_{i}=\operatorname{round}\left(\frac{d_{A B C D}-\gamma_{i}}{\lambda_{i}}\right) \cdot \lambda_{i}
$$

represents the best fit given q-range $d_{A B C D}$; and $\gamma_{i}$ represents the phase offset relative to the wavelength $\gamma_{i}=\lambda_{i} \frac{\Delta \varphi_{i}}{2 \pi}$.

Most existing RIP algorithms consider the value of $d_{A B C D}$ that minimizes Equation 3 as the q-range estimate $\left(\min \left(E R R O R\left(d_{A B C D}\right)\right)\right.$, e.g. [2]). Figure 4 shows Equation 3 as a function of the q-range. The vertical red line represents the real q-range. Note that the real q-range lies in a local optimum, which is a known problem of the RIP system ([3]). Also note that Equation 3 is periodic with a relatively high frequency, which explains that the global minimum is near the global maximum.

Existing RIP implementations distinguish between two type of nodes, namely:

- Reference nodes know their position in advance.

- Blind nodes do not know their location in advance and are subject to localization.

Existing RIP implementations assume that two transmitting and one receiving node are reference nodes. In this case we assume that nodes $A / B / C$ are reference nodes and node $D$ is a blind node. This means that the values of $d_{A C}$ and $d_{B C}$ are known and that the values of $d_{A D}$ and $d_{B D}$ are unknown. Then, Equation 2 can be rewritten as follows:

$$
d_{A B C D}+d_{A C}-d_{B C}=d_{A D}-d_{B D}
$$

Note that the value of the left hand side of Equation 4 is known $\left(d_{A B C D}+d_{A C}-d_{B C}\right)$. Equation 4 represents a hyperbolic curve over the localization surface as with TDOA measurements ([1]). The intersection of two or more hyperbolic curves represents the position, assuming perfect q-range estimates. This means that RIP requires frequency beat measurements between two or more different pairs of senders in order to estimate the position. Figure 2 shows an example of two hyperbolic curves $\left(H_{A B}\right.$ and $\left.H_{A C}\right)$ calculated by the following sender pairs: $\{A, B\}$ and $\{A, C\}$. The circles represent the positions of the reference nodes $A, B$ and $C$. The triangle represents the position of node $D$, the position of which is estimated by the intersection of the hyperbolic curves. Unfortunately, q-range measurements contain error, 


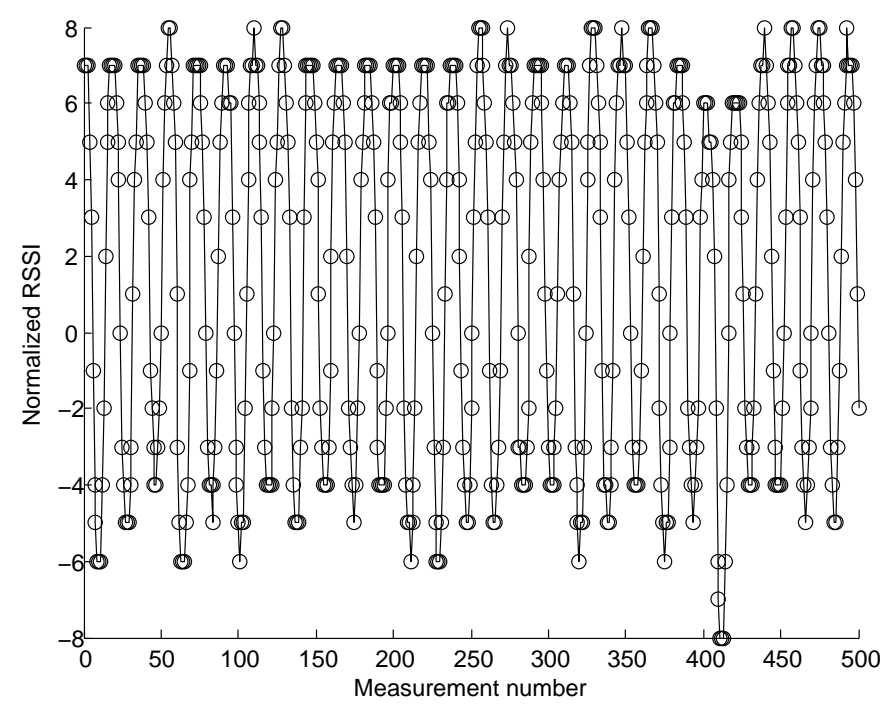

Fig. 3. Frequency beat measurement

and the position can only be estimated. The position estimate is defined by the following optimization function (e.g. [2] and [8]):

$$
\min _{\hat{x}, \hat{y}} \sum_{j=1}^{K}\left(d_{A B C D, j}-d_{A B C D}\right)^{2}
$$

Here $\hat{x}$ and $\hat{y}$ represent the $\mathrm{x}$ - and $\mathrm{y}$-coordinate of the position estimate; $K$ represents the total number of q-ranges; $d_{A B C D, j}$ represents the $j$ 'th q-range calculated by minimizing Equation 3 ; and $d_{A B C D}$ represents the q-range to position estimate $(\hat{x}, \hat{y})$.

[2] introduces RIP and provides a detailed analysis and description of the implementation of RIP on a CC1000 platform. Existing work on RIP extends [2] by - increasing its scalability to position any number of nodes ([3]) - decreasing the required computational costs ([4]) - making RIP suitable for localization of mobile blind nodes ([5]) - optimally choosing sender pairs $([8]) .[6] /[7] /[9][10]$ measure the Doppler effect using frequency beat measurements. This information is used to locate mobile nodes. In this paper, we focus on the localization of static nodes.

\section{ERROR SOURCES}

This section analyzes the sources of error caused by the radios using the RIP method. The results of this section are used to compare and analyze the errors introduced by the CC2430 and CC1000 platform. This section shows that the phase measurement error increases with the frequency of the measured frequency beat $\left(\left|f_{a}-f_{b}\right|\right)$.

Throughout this section we consider the errors introduced by the radios performing one phase offset measurement $\left(\Delta \varphi_{i}\right.$ in Equation 1). One phase offset measurement involves two nodes transmitting unmodulated carriers and two receivers measuring the phases of the received signal amplitudes, as illustrated in Figure 1. The sampling rate of the RSSI determines whether

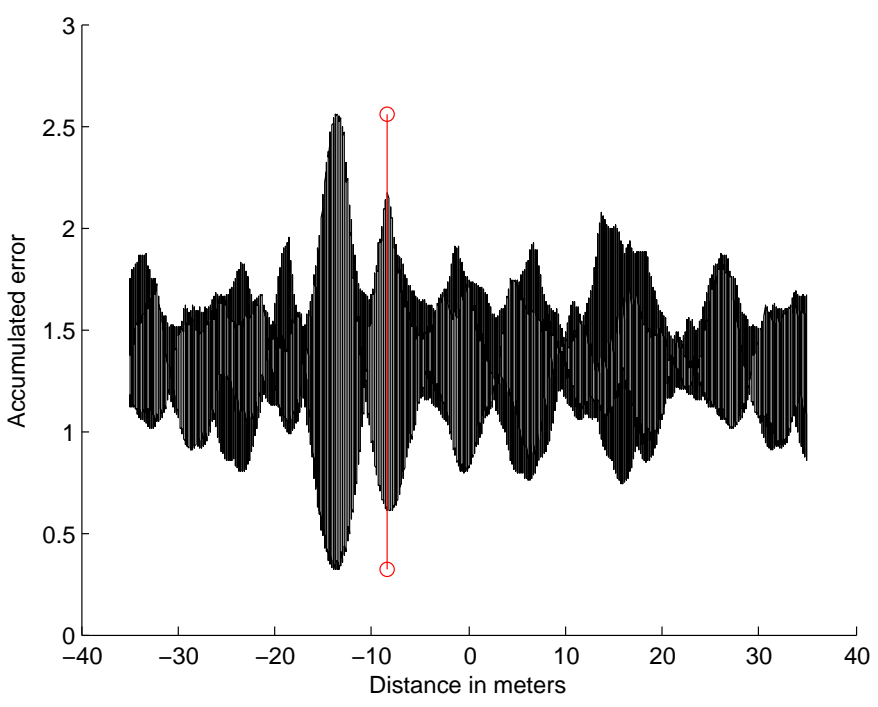

Fig. 4. Q-range error distribution with multipath effect

the frequency beat is measurable or not. The sources of error caused by the radio are the following ([2]):

- The time synchronization error between the receivers introduces the following phase measurement errors:

$$
\varphi_{\text {error } \text { sync }} \approx 2 \pi \cdot \operatorname{sync}_{a c c} \cdot\left|f_{a}-f_{b}\right|
$$

Here $s_{n n c_{a c c}}$ represents the synchronization accuracy in seconds. This equation only holds when:

$$
\text { sync }_{a c c}<\frac{1}{\left|f_{a}-f_{b}\right|}
$$

- The RSSI measurement time error of the receivers introduces the following phase measurement errors:

$$
\varphi_{\text {error }_{\text {meas }}} \approx 2 \pi \cdot \frac{\text { meas }_{a c c}}{2} \cdot\left|f_{a}-f_{b}\right|
$$

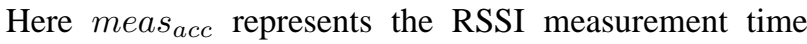
error in seconds over the total measurement time. This equation only holds when:

$$
\frac{\text { meas }_{a c c}}{2}<\frac{1}{\left|f_{a}-f_{b}\right|}
$$

- The carrier frequency inaccuracy of the senders introduces the following phase measurement errors:

$$
\varphi_{\text {error }_{c a r}} \approx 2 \pi\left|\frac{d_{A B C D}}{c / f_{i}}-\frac{d_{A B C D}}{c /\left(f_{i}+f_{o f f}\right)}\right|
$$

Here $f_{\text {off }}$ represents the frequency offset in Hertz.

- The frequency drift of the transmitted signal. We assume that the frequency drift has a negligible influence on the phase due to the relatively short measurement time, as in [2].

- The phase noise of the transmitted signal. [2] assumes that the phase noise has a negligible influence on the phase. The $\mathrm{CC} 2430$ has a lower phase noise than the $\mathrm{CC} 1000$, therefore we also assume that the phase noise has a negligible influence on the phase. 

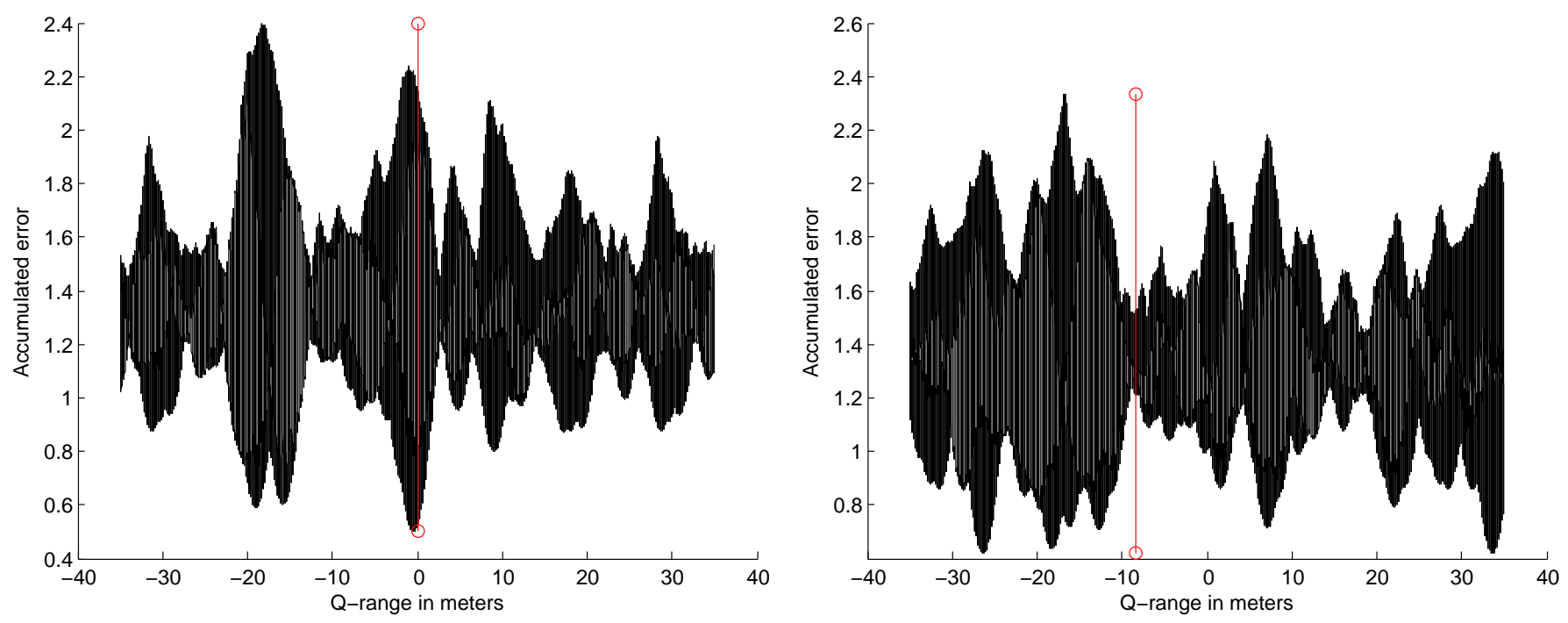

Fig. 5. Q-range error distribution of good combination of senders and receivers Fig. 6. Q-range error distribution of bad combination of senders and receivers

The calculated phase errors are not independent, because we expect that the errors remain largely similar over the frequencies for every sender pair. In other words, the errors are biased. Therefore, we do not know the influence of these errors on the final position estimate. We leave this topic open for future research.

This enumeration verifies that the phase measurement error increases with the frequency of the measured frequency beat $\left(\left|f_{a}-f_{b}\right|\right)$. However, a lower frequency beat increases the required measurement time and thus increases the phase measurement error caused by the frequency drift. The next section describes how the existing and new implementation deal with this problem.

\section{Existing AND New ImPlementation Strategy}

This section first analyzes the existing implementation strategy on the CC1000 platform. After that, it analyzes the errors introduced by a RIP implementation on a CC2430 platform. This section compares both implementations and shows that the CC2430 platform increases the errors in comparison with the CC1000 platform.

\section{A. CC1000 Platform}

Section III showed that most error sources grow linearly with the frequency beat $\left(\left|f_{a}-f_{b}\right|\right)$. The existing RIP implementation minimizes the frequency separation and thus the phase measurement errors by introducing a frequency tuning phase. Since the measurable frequencies are limited by the measurement time of $29 \mathrm{~ms}$ as well as the limited RSSI sampling rate of $9 \mathrm{kHz}$, the frequency tuning phase calibrates the frequency separation for every pair of senders between the 200 and $800 \mathrm{~Hz}$ ([2]). The frequency tuning phase requires a radio that has the ability to tune its frequency in fine-grain steps smaller than the wanted frequency separation, like the CC1000 (see [11], 65 Hertz frequency resolution). This approach minimizes the phase measurement errors. For a detailed description of the requirements and how the existing RIP implementation complies with these requirements, we refer to [2].

\section{B. CC2430 Platform}

The frequency resolution of the CC2430 is $1 \mathrm{MHz}$, which is not sufficient for a frequency tuning phase. Without a frequency tuning phase, the frequency separation depends on the difference between the two crystal oscillators. The maximum frequency separation depends on the crystal accuracy (+/- 40 $\mathrm{ppm})$ and the maximum frequency of the unmodulated carrier signal $(2.48 \mathrm{GHz})$ :

$$
\max \left(\left|f_{a}-f_{b}\right|\right)=2 \cdot \frac{40}{1000000} \cdot 2.48 \mathrm{GHz}=198400 \mathrm{~Hz}
$$

The RSSI sampling rate of the CC2430 equals $62.5 \mathrm{KHz}$. This means that not every frequency beat is measurable on a CC2430 platform. In this paper, we randomly picked 4 CC2430 radios that all had a measurable frequency beat $(\sim$ $2 \ldots \sim 7 \mathrm{kHz}$.). A possible explanation for these measurable frequency beats is that the crystal oscillators are all from the same batch. The sources of error caused by the CC2430 are the following (see Section III):

- The time synchronization error. The time synchronization error introduced by the the CC2430 depends on the accuracy of the crystal oscillator, because the MAC controller enables clock synchronization accuracy. The time between the synchronization message and the start of the RSSI measurements $(1.4 \mathrm{~ms})$ determines the synchronization accuracy. In our case this equals:

$$
\text { sync }_{a c c} \approx \frac{40}{1000000} \cdot 1.4 \mathrm{~ms}=56 \mathrm{~ns}
$$

Then the maximum phase measurement error caused by the time synchronization error equals (using Equation 6):

$$
\varphi_{\text {error }}^{\text {sync }} \approx 1.1 \% \cdot 2 \pi
$$




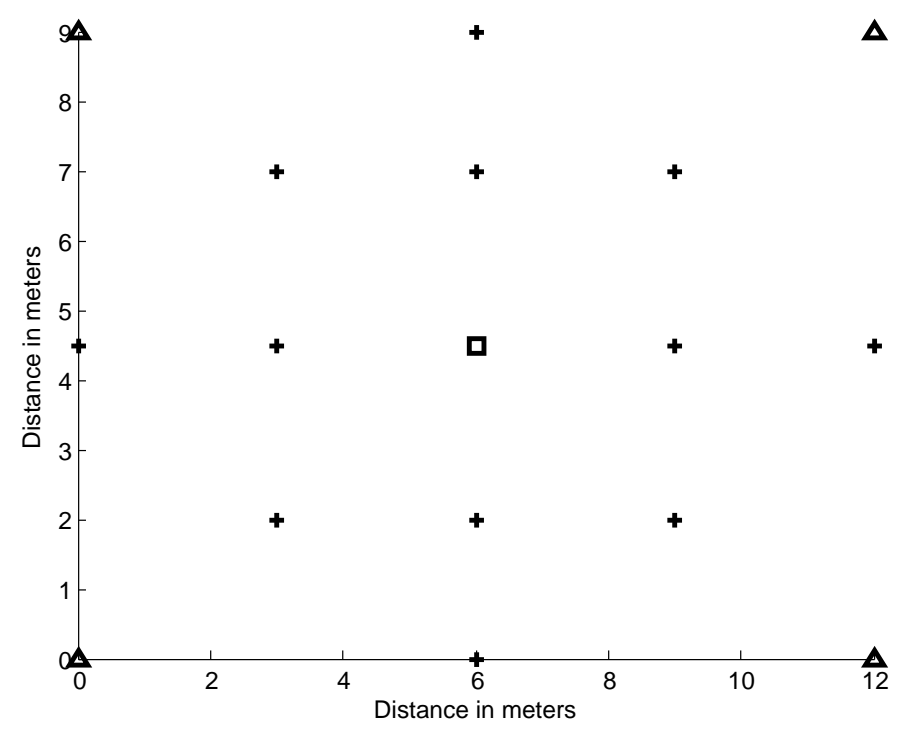

Fig. 7. Measurement set-up

- The RSSI measurement time error. The RSSI measurement time error introduced by the CC2430 depends on the accuracy of the crystal oscillator, because the DMA controller enables clock accuracy. Therefore, the total RSSI measurement time $(8 \mathrm{~ms})$ determines the RSSI measurement time accuracy. In our implementation this equals:

$$
\text { meas }_{a c c} \approx \frac{40}{1000000} \cdot 8 \mathrm{~ms}=320 \mathrm{~ns}
$$

Then the maximum phase measurement error caused by the RSSI measurement time error equals (using Equation 7):

$$
\varphi_{\text {error }}^{\text {meas }} \approx 3.2 \% \cdot 2 \pi
$$

- The carrier frequency inaccuracy. The maximum frequency offset depends on the crystal accuracy and equals $99.2 \mathrm{kHz}$. Then the maximum phase measurement error caused by the carrier frequency inaccuracy equals $\left(d_{A B C D}=100\right.$ meter, using Equation 8):

$$
\varphi_{\text {error }} \approx 3.3 \% \cdot 2 \pi
$$

The calculated phase measurement errors represent the maximum phase measurement error per receiver. The maximum error of the relative phase offset is approximately twice the maximum phase measurement error per receiver. Therefore, we expect that the hardware has a significant influence on the accuracy of the phase measurement and thus the accuracy of the estimated q-range. This means that the phase measurement error depends on the combination of the used senders and receivers. This error reduces as the number of phase measurements increases, however the sender/receiver dependent bias remains (see Section III). Section V further analyzes these type of dynamic errors.

This reasoning also shows that the phase measurement accuracy increases linearly with the accuracy of the crystal oscillator. Therefore, replacing the existing crystal oscillator with a more accurate one increases the accuracy of the phase measurements.

Table I summarizes the results of this section and shows that the CC1000 platform is significantly better suited for RIP localization as:

- The maximum time synchronization error is an order of magnitude smaller with the $\mathrm{CC} 1000\left(\varphi_{\text {error }_{\text {sync }}} \approx 0.2 \%\right)$

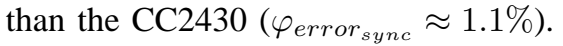

- The maximum RSSI measurement time error is an order of magnitude smaller with the CC1000 $\left(\varphi_{\text {error }}\right.$ meas $\approx$

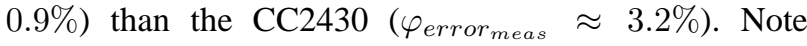
that [2] does not provide any details about the RSSI measurement time error, therefore we assume that the CC1000 has a similar RSSI measurement time jitter as the CC2430.

- The maximum carrier frequency inaccuracy is an order of magnitude smaller with the $\mathrm{CC} 1000\left(\varphi_{\text {error }}\right.$ acc $\approx$ $0.04 \%)$ than the $\mathrm{CC} 2430\left(\varphi_{\text {error }_{a c c}} \approx 3.3 \%\right)$ at a distance of 100 meters.

Therefore, we expect that RIP localization with the CC2430 platform decreases the performance compared to the $\mathrm{CC} 1000$ platform. The advantage of the CC2430 platform is that it decreases the measurement time significantly.

\begin{tabular}{|l|l|l|}
\hline Platform & CC1000 & CC2430 \\
\hline Accuracy & Frequency calibration & Crystal oscillator \\
Synchronization error & $2 \mu \mathrm{s}$. & $56 \mathrm{~ns}$ \\
Measurement time & $28.4 \mathrm{~ms}$. & $8 \mathrm{~ms}$ \\
RSSI sampling rate & $9 \mathrm{kHz}$. & $62.5 \mathrm{kHz}$. \\
Frequency beat & $200 \ldots 800 \mathrm{~Hz}$. & $\sim 2 \ldots \sim 7 \mathrm{kHz}$. \\
Frequency inaccuracy & $2 \mathrm{kHz}$. & $99.2 \mathrm{kHz}$. \\
Frequency range & $400-800 / 900 \mathrm{MHz}$ & $2.4 \mathrm{GHz}$. \\
\hline
\end{tabular}

TABLE I

PLATFORM CHARACTERISTICS 


\section{Measurement Set-up and Performance EVALUATION}

This section first provides a description of the measurement and localization set-up. After that, this section evaluates the performance of the existing RIP algorithm and an initial implementation of a new RIP algorithm.

\section{A. Measurement Set-up}

Figure 7 shows the measurement set-up used throughout this paper. Here the four triangles and one square represent the reference node locations; the crosses represent the blind node locations. The difference between the reference nodes (triangles and square) is that the reference node represented by the triangle is only used for measuring the frequency beat. The measurements were conducted in a $12 \times 9$ meter indoor environment with six CC2430 radio's ([12]). We used five CC2430 radio's as reference nodes; these reference nodes were static during and between the measurement rounds (triangles and square). We used one CC2430 radio as blind node; this blind node measured the RSS at 12 different locations (crosses) relative to the reference nodes. The blind node measured 500 consecutive RSS measurements over $8 \mathrm{~ms}$ per frequency over a total of 16 frequencies in a bandwidth of $2405 \ldots 2480 \mathrm{MHz}$. The nodes all had a widely used "omnidirectional" dipole antenna with a vertical orientation. The radios were all placed at the same height at 1.5 meter in order to minimize noise (e.g. [3]). All individual RSS measurements were sent to a computer and logged for post processing. The conditions during the measurements were static (temperature, humidity, no moving objects). Therefore, we consider this environment as a static environment.

\section{B. Sources of error}

We measured and analyzed the following sources of error:

- Multipath effects cause q-range estimate ambiguity, as illustrated by Figure 4. Figure 4 shows that the q-range (red vertical line) is at a local minimum instead of the global minimum. [3] tries to solve this problem by iteratively limiting the search space of the q-range. The problem with this approach is that it assumes a priori knowledge about the phase measurement error. Moreover, it assumes that the global minimums provide some knowledge about the real q-ranges. The problem is that we do not have this information available using the CC2430 platform.

- The accuracy depends on the combination of senders and receivers, which is a problem with the C2430 platform as outlined in Section IV. A good combination of senders and receivers tend to have the lowest minimum at the real q-range. Figure 5 shows a typical example of a q-range distribution with a good combination of senders and receivers.

A bad combination of senders and receivers tend to have a lot of peaks in the q-range error distribution. Moreover, the differences between the peaks tend be smaller. Figure
6 shows a typical example of a q-range distribution with a bad combination of senders and receivers.

The initial implementation of our new algorithm uses this information to weight the individual measurements accordingly and enhances the position estimate.

\section{Performance Evaluation}

Table II shows the performance of the existing RIP algorithm and an initial implementation of a new algorithm. The numbers in the first column represent the position of the blind node; the numbers in the second and third column represent the localization error in meters per localization algorithm. This table shows that the new algorithm decreases the mean error from 3.1 to 1.5 meter and the median error from 2.8 to 1.0 meter. Moreover, this algorithm provides better results than the RIP algorithm in eleven of the twelve cases.

\begin{tabular}{|l|l|l|}
\hline position / algorithm & RIP & Initial implementation \\
\hline$(0,4.5)$ & $4.5 \mathrm{~m}$. & $0.1 \mathrm{~m}$. \\
$(6,0)$ & $0.25 \mathrm{~m}$. & $0.1 \mathrm{~m}$. \\
$(6,9)$ & $0.9 \mathrm{~m}$. & $2.9 \mathrm{~m}$. \\
$(12,4.5)$ & $0.25 \mathrm{~m}$. & $0.2 \mathrm{~m}$. \\
$(6,7)$ & $3.2 \mathrm{~m}$. & $0.3 \mathrm{~m}$. \\
$(3,4.5)$ & $1.8 \mathrm{~m}$. & $0.3 \mathrm{~m}$. \\
$(6,2)$ & $2.4 \mathrm{~m}$. & $2.3 \mathrm{~m}$. \\
$(9,4.5)$ & $6.3 \mathrm{~m}$. & $5.3 \mathrm{~m}$. \\
$(3,7)$ & $8.8 \mathrm{~m}$. & $1.1 \mathrm{~m}$. \\
$(3,2)$ & $2.5 \mathrm{~m}$. & $0.9 \mathrm{~m}$. \\
$(9,2)$ & $3.6 \mathrm{~m}$. & $1.9 \mathrm{~m}$. \\
$(9,7)$ & $3.0 \mathrm{~m}$. & $2.1 \mathrm{~m}$. \\
\hline MEAN ERROR: & $3.1 \mathrm{~m}$. & $1.5 \mathrm{~m}$. \\
MEDIAN ERROR: & $2.8 \mathrm{~m}$. & $1.0 \mathrm{~m}$. \\
\hline \multicolumn{3}{|c|}{ TABLE II } \\
\hline \multicolumn{3}{|c|}{ LOCALIZATION PERFORMANCE }
\end{tabular}

\section{CONCLUSION}

In this study, we evaluated and implemented RIP on a 2.4 $\mathrm{GHz}$ radio platform, using IEEE 802.15.4. We evaluated this by performing real measurements in an indoor environment. These measurements show that the existing RIP algorithm does not provide the required accuracy, because it cannot cope with the large and dynamic sources of error that are incurred with the novel type of radios. An initial implementation of an improved RIP shows that the accuracy of the standard RIP can be improved significantly, and is able to cope with the dynamic errors. The main conclusion of this paper is that we have shown that a RIP system can be implemented on other platforms than the $\mathrm{CC} 1000$ and provides a reasonable lcoalization performance. We expect that the performance can be increased significantly by increasing the accuracy of the crystal and by increasing the number of frequency beat measurements.

Future work will evaluate this new algorithm in depth, and evaluate the performance using a more accurate crystal and a larger amount of frequency beat measurements in a larger area. 


\section{ACKNOWLEDGEMENTS}

We would like to thank the reviewers for their constructive feedback.

This paper describes work in part undertaken in the context of the TSP project. The TSP project has joint funding from the European Regional Development Fund of the European Union and the Dutch Provinces of Gelderland and Overijssel.

\section{REFERENCES}

[1] N.Patwari: Location estimation in sensor networks. Thesis of Neal Patwari at University of Michigan, 2005.

[2] M.Marti, P.Vlgyesi, S.Dra, B.Kusy, A.Ndas, .Ldeczi, G.Balogh, K.Molnr: Radio interferometric geolocation. SenSys 2005: pp. 1-12.

[3] B.Kusy, .Ldeczi, M.Maroti, L.G.L.T.Meertens: Node density independent localization. IPSN 2006: 441-448.

[4] B.Kusy, J.Sallai: Analytical solution for radio-interferometric localization of mobile sensors. ISIS technical report, ISIS-06-710. May 2006.

[5] B.Kusy, G.Balogh, J.Sallai, .Ldeczi, M.Marti: InTrack: High Precision Tracking of Mobile Sensor Nodes. EWSN 2007: 51-66.

[6] B.Kusy, J.Sallai, G.Balogh, .Ldeczi, V.Protopopescu, J.Tolliver, F.DeNap, M.Parang: Radio interferometric tracking of mobile wireless nodes. MobiSys 2007: 139-151.

[7] B.Kusy, .Ldeczi, X.D.Koutsoukos: Tracking mobile nodes using RF Doppler shifts. SenSys 2007: 29-42.

[8] H.Wu, H.Chang, C.You, H.Chu, P.Huang: Modeling and optimizing positional accuracy based on hyperbolic geometry for the adaptive radio interferometric positioning system. International Symposium on Location- and Context-Awareness (LOCA 2007), Oberpfaffenhofen, Germany, September 2007, pp 228-244.

[9] J.Tian, H.Chang, T.Lai, H.Chu: SpinTrack: Spinning Infrastructure Nodes for Precise Indoor Localization. In the poster session of UBICOMP, September 2008.

[10] H.Chang, J.Tian, T.Lai, H.Chu, P.Huang: Spinning Beacons for Precise Indoor Localization. ACM SENSYS 2008, November 2008.

[11] http://focus.ti.com/lit/ds/symlink/cc1000.pdf, 2010.

[12] http://focus.ti.com/lit/ds/symlink/cc2430.pdf, 2010. 